\title{
Cryptococcose neuroméningée compliquée d'hydrocéphalie chez un enfant VIH négatif.
}

\section{Neuromeningeal cryptococcosis complicated by hydrocephalus in an HIV negative child.}

Doumbia $\mathrm{AK}^{1}$,Togo $\mathrm{P}^{1}$,Coulibaly $\mathrm{O}^{1}$,Dembélé $\mathrm{A}^{1}$, Kané $^{2}$,Diakité $\mathrm{AA}^{1}$.

1. Département de Pédiatrie du CHU Gabriel Touré

2. Service de pédiatrie Hôpital du Mali

Auteur correspondant : Abdoul Karim Doumbia, Enseignant-chercheur au département de pédiatrie CHU Gabriel Touré - Bamako (Mali). Email : doumbiav@gmail.com.

\section{RESUME}

La cryptococcose neuroméningée (CNM) est une mycose cosmopolite grave, affectant généralement les sujets ayant un déficit de l'immunité cellulaire surtout les séropositifs au virus de l'immunodéficience humaine (VIH).Nous rapportons l'observation d'un cas chez une fille de 7 ans, admise pour altération de l'état général, trouble de la conscience (Glasgow à 8/15), syndrome méningé (raideur de la nuque, signe de Kernig et de Brudzinski) et tétraplégie prédominant à gauche. La tomodensitométrie (TDM) cérébrale avait mis en évidence une dilatation de l'ensemble des cavités ventriculaires avec hypodensité périventriculaire cérébrale.La ponction lombaire (PL) avait ramené un liquide cérébrospinal (LCS) clair. L'analyse du LCS avait permis de retrouver une méningite pléiocytaire, hyperprotéinorachique, hypoglycorachique et un Cryptococcusneoformans. Un traitement antifungique associant amphotéricine B et fluconazole avait été instauré.La patiente avait aussi subit un drainage chirurgical de l'hydrocéphalie. Après 12 semaines d'hospitalisation, le décès était survenu dans un contexte d'altération de l'état général, de convulsions à répétition et de coma profond. La cryptococcose neuroméningée pose un sérieux problème de diagnostic et de traitement chez l'enfant immunocompétent à cause de l'insuffisance des moyens d'investigation et de traitement.

\section{ABSTRACT}

Neuromeningeal cryptococcosis (CNM) is a serious cosmopolitan mycosis, generally affecting people with a deficient cellular immunity, especially those who are seropositive to the human immunodeficiency virus (HIV). We report the observation of a case in a 7-year-old girl, admitted for deterioration of general condition, disorder of consciousness (Glasgow at 8/15), meningeal syndrome (stiffness of the neck, Kernig's sign and Brudzinski) and quadriplegia predominantly on the left. Computed tomography (CT) of the brain showed dilation of all the ventricular chambers with cerebral periventricular hypodensity. The lumbar puncture (PL) brought back clear cerebrospinal fluid (CSL). LCS analysis found pleocytic, hyperproteinorachic, hypoglycorachic meningitis and Cryptococcus neoformans. Antifungal therapy combining amphotericin B and fluconazole had been initiated. The patient also had surgical drainage for hydrocephalus. After 12 weeks of hospitalization, death occurred in the context of deterioration of general condition, recurrent convulsions and deep coma.Neuromeningeal cryptococcosis poses a serious problem of diagnosis and treatment in immunocompetent children because of insufficient means of investigation and treatment.

\section{INTRODUCTION}

La cryptococcose neuroméningée est une infection mycosique grave touchant essentiellement le sujet immunodéprimé et en particulier, le sujet séropositif au VIH [1]. C'est une mycose cosmopolite due à une levure encapsulée (Fungi) appartenant à la famille des Tremellaceae. Il existe trois types de C. neoformans: C. neoformansvar. neoformans(sérotype $\mathrm{D}), \quad C$. neoformansvar. gattii(sérotypes $\mathrm{B}-\mathrm{C}$ ) et $C$. neoformansvar.grubii(sérotype A) qui sont pathogènes pour l'homme [2]. Le cryptocoque vit à l'état libre en saprophyte dans la nature et dans le sol enrichi en matières organiques [1]. Il a une prédilection pour le tissu nerveux. L'infection par le virus de l'immunodéficience humaine (VIH) est le principal facteur prédisposant à la cryptococcose. En France, $80 \%$ des cas sont observés chez des individus infectés par le VIH [3]. En absence de SIDA, il faut éliminer une corticothérapie au long cours, une hémopathie lymphoïde, un diabète, une insuffisance rénale ou une cirrhose [1]. La forme clinique la plus fréquente et la plus grave est la méningo-encéphalite [4]. La majorité des rares cas rapportés chez l'enfant par la littérature ont presque tous eu une évolution péjorative [7]. A l'échelle mondiale, environ 957900 cas de cryptococcose neuroméningée surviennent chaque année, entrânant 624700 décès dans les 3 mois suivant l'infection [1]. L'intérêt de cette présentation est de rapporter un cas de cryptococcose neuroméningée chez l'enfant sans signe d'immunodépression et de faire une mise au point sur cette pathologie en pédiatrie.

\section{PATIENT ET OBSERVATION}

Il s'agissait d'une fillette de 7 ans hospitalisée pour des céphalées intenses associées à des vomissements en jet et une raideur de la nuque, d'installation progressive, évoluant depuis 3 mois dans un contexte fébrile. L'examen physique avait permis de retrouver une altération de l'état général, un trouble de la conscience (Glasgow à 8/15), un 
syndrome méningé franc (raideur de la nuque, signe de Kernig et de Brudzinski), une vivacité anormale des réflexes ostéotendineux et une tétraplégie prédominant à gauche. Par ailleurs, on notait une dysarthrie, une agitation par moment et des crises d'épilepsie intermittentes.

Les examens de laboratoire avaient permis de retrouver une hyperleucocytose $(13800 \mathrm{Giga} / \mathrm{l})$ à prédominance neutrophiles (76\%), une anémie modérée $(10 \mathrm{~g} / \mathrm{dl})$.La TDM cérébrale avait permis d'objectiver une dilatation de l'ensemble des cavités ventriculaires, une hypodensité périventriculaire cérébrale compatible avec une résorption trans-épendymaire et une hydrocéphalie [figure 1].

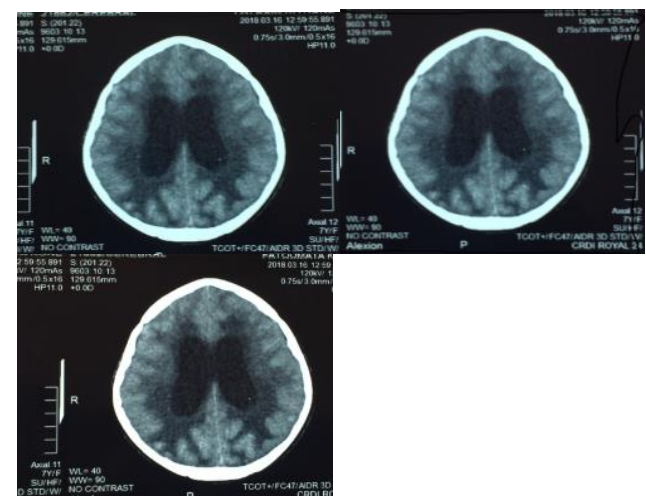

$\underline{\text { Figure } 1}$ : Images du scanner cérébral de la patiente montrant une hydrocéphalie tétra-ventriculaire.

La PL avait ramené un LCS clair avec 40 leucocytes $/ \mathrm{mm} 3$. L'examen direct était négatif et l'agglutination n'avait pas été faite. La patiente avait été mise sous ceftriaxone (100mg/kg par jour) et gentamicine $(3 \mathrm{mg} / \mathrm{kg} / \mathrm{jour})$ pendant une semaine. Devant l'aggravation des signes neurologiques, une seconde ponction lombaire avait ramené un LCS clair dont l'analyse avait permis de retrouver une méningite pléiocytaire, hyperprotéinorachique, hypoglycorachique. L'étude mycologique du LCS avait montré à l'examen direct à l'encre de chine des levures encapsulées. La culture avait été positive

identifiantCryptococcusneoformans. L'électroencép halogramme avait montré des ondes lentes diffuses, traduisant une souffrance encéphalique. La sérologie VIH était négative à deux reprises et le taux de CD4 était normal. Le diagnostic de CNM compliquée d'hydrocéphalie ayant été retenu, le patient avait étémis sous fluconazole(à la dose de $12 \mathrm{mg} / \mathrm{kg} / \mathrm{j}$ pendant 14 jours) et amphotéricine B (à $1 \mathrm{mg} / \mathrm{kg} /$ jour per os). L'hydrocéphalie avait nécessité un drainage chirurgical.Le décès était survenu dans un contexte d'altération de l'état général, de convulsions à répétition et de coma profond.

\section{DISCUSSION}

La cryptococcose neuroméningée est une mycose opportuniste grave du système nerveux central causée par un champignon encapsulé de type levure, Cryptococcusneoformans. Le fardeau lié à cette affection est très lourd dans les pays à revenu faible et moyen où l'incidence du virus de l'immunodéficience humaine (VIH) est très élevée $[2,4,8]$. La suspicion clinique d'infection cryptococcique se limite essentiellement aux patients immunodéprimés, ce qui sous-estime la fréquence de la maladie [8]. En dehors du VIH, il est important d'identifier d'autres comorbidités telles que la malnutrition ou la chimiothérapie (corticostéroïdes, agents cytotoxiques et anticorps monoclonaux) [5,6]. Au cours des 15 dernières années, des études ont mis en évidence un groupe de patients atteints de troubles immunologiques primaires inconnus prédisposant à l'infection cryptococcique [5]. Ces pseudo-patients immunocompétents présentent des problèmes diagnostiques majeurs. Dans cette observation, la patiente ne présentait aucun facteur prédisposant connuet les signes cliniques retrouvés (fièvre, raideur de la nuque, céphalées et déficit neurologique) étaient similaires à ceux généralement décrits dans la littérature $[1,8,9,10]$. Le diagnostic de CNM repose sur l'examendirect du LCS après coloration par l'encre de Chine. L'examen cyto-chimique du LCS montre presque toujours une cellularité modérément élevée à prédominance lymphocytaire, une hypoglycorrachie et une hyperprotéinorrachie $[1,5]$. Les techniques de diagnostic indirect sont essentiellement représentées par la recherche d'antigène cryptococcique (cryptolatex), qui est positif dans $90 \%$ des LCS et $70 \%$ des sérums de patients avec cryptococcose neuro-méningée [7,10]. Dans la cryptococcose neuroméningée du sujet VIH négatif, le taux de leucocytes et des protéines du LCS sont plus élevés et la glycorrachie est inférieure à celui des patients infectés par le VIH [5]. Le bilan radiologique de la CNM est indispensable et permet d'évaluer le pronostic initial et de guider le choix $\mathrm{du}$ protocole thérapeutique [10].L'aspect tomodensitométrique de la CNM est extrêmement polymorphe, sans aucune spécificité. L'atteinte neuroméningée se complique fréquemment d'hypertension intracrânienne $(50 \%)$, définie par une pression intracrânienne supérieure à $200 \mathrm{mmHg}$ en décubitus latéral [8]. L'IRM cérébrale est en règle normale mais a l'intérêt d'éliminer les autres diagnostics. La dilatation des espaces de Virchow Robin est très évocatrice de la cryptococcose [10]. La CNM chez les sujets VIH négatifs constitue une urgence thérapeutique. L'arsenal antifongique actuellement disponible pour le traitement de la cryptococcose est limité à trois médicaments, utilisés seuls ou en association: l'amphotéricine $\mathrm{B}(0,7$ à $1 \mathrm{mg} / \mathrm{kg} / \mathrm{j})$, flucytosine $(5$ fluorocytosine ou $5-\mathrm{FC})(100 \mathrm{mg} / \mathrm{kg} / \mathrm{j})$ pendant deux semaines et fluconazole $(400 \mathrm{mg} / \mathrm{j}$ en une prise orale) pendant 8 semaines au minimum ou jusqu'à 
stérilisation des cultures mycologiques [7, 8, 9]. Le traitement des infections du SNC est souvent difficile car la barrière hémato-encéphalique (BHE) limite la diffusion des molécules vers les tissus cérébraux [8]. Le traitement de la méningite à cryptocoques comprend trois phases: l'induction (2 semaines), la consolidation (8 semaines) et l'entretien (6 à 12 mois). Les directives de la Society for InfectiousDiseases of America et de l'Organisation mondiale de la santé soulignent l'importance de l'utilisation de médicaments fongicides puissants (5-fluorocytosine ou 5FC) pendant la phase d'induction; cependant, l'accès mondial aux médicaments antifongiques est encore insuffisant, ce qui souligne l'importance des stratégies de traitement alternatives[8]. L'issuefatale de cette patiente était prévisible compte tenu de l'existence de plusieurs facteurs de mauvais pronostic (le retard diagnostique, la dénutrition et l'indisponibilité d'un traitement adapté).

\section{CONCLUSION}

La cryptococcose neuro-méningée est une maladie opportuniste sous-diagnostiquée chez l'enfant VIH négatif en atteste la rareté des cas décrits dans la littérature. Elle est généralement responsable d'une dont le pronostic est péjoratif en l'absence d'un diagnostic précoce et d'un traitement adéquat et urgent.

Conflit d'intérêt : aucun

\section{REFERENCES}

1. Abassi M, Boulware DR, Rhein J. Cryptococcal Meningitis: Diagnosis and Management Update. Curr Trop Med Rep. 2015 Jun 1;2(2):90-99.
2. Arif S, Ghazanfar K, Muhammad WW, Malik H. Cryptococcal meningitis in immunocompetent patient. J Ayub Med Coll Abbottabad. 2015 OctDec;27(4):942-4.

3. Recio R, Perez-Ayala A. Cryptococcus neoformans Meningoencephalitis. N Engl J Med. 2018 Jul 19;379(3):281.

4. Chrétien F, Lortholary O, Kansau I, Neuville S, Gray F, Dromer F. Pathogenesis of cerebral Cryptococcus neoformans infection after fungemia. J Infect Dis. 2002 Aug 15;186(4):522-30.

5. Shribman S, Noyce A, Gnanapavan S, Lambourne J, Harrison T, Schon F. Cryptococcal meningitis in apparently immunocompetent patients: association with idiopathic CD4+ lymphopenia. Pract Neurol. 2018 Apr;18(2):166-169.

6. Ndiaye M, Diagne NR, Seck LB, Sow AD, Sène MS, Diop AG, Sow HD, Ndiaye MM: Cryptococcal meningitis in children: description of 3 cases. Med Trop (Mars). 2011,71(2):176-178.

7. Artru P, Schleinitz N, Gauzere BA, Artru S, Paganin F, Roblin X. Cryptococcose neuroméningée et cirrhose alcoolique. Gastroenterol Clin Biol. 1997;21(1):78-81. French.

8. Spadari CC, Wirth F, Lopes LB, Ishida K. New Approaches for Cryptococcosis Treatment. Microorganisms. 2020 Apr 23;8(4):613.

9. Soumaré M, Seydi M, Ndour CT, Dieng Y, Diouf AM, Diop BM. Aspects actuels de la cryptococcose neuro-méningée à Dakar. Med Trop. 2005;65:559-60.

10. Oumar AA, Dao S, Ba M, Poudiougou B, Diallo A. Aspects épidémiologique, clinique et pronostique de la cryptococcose neuroméningée en milieu hospitalier de Bamako, Mali. Rev Med Brux. 2008;29:145-216. 